\title{
Role of Particle Shape on the Stress Propagation in Granular Packings
}

\author{
Raúl Cruz Hidalgo, ${ }^{1}$ Iker Zuriguel, ${ }^{2}$ Diego Maza, ${ }^{2}$ and Ignacio Pagonabarraga ${ }^{3}$ \\ ${ }^{1}$ AMADE, Departament de Física, Universitat de Girona, 17071 Girona, Spain \\ ${ }^{2}$ Departamento de Física, Facultad de Ciencias, Universidad de Navarra, 31080 Pamplona, Spain \\ ${ }^{3}$ Departament de Física Fonamental, Universitat de Barcelona, 08028 Barcelona, Spain \\ (Received 13 May 2009; revised manuscript received 3 August 2009; published 9 September 2009)
}

\begin{abstract}
We present an experimental and numerical study on the influence that particle aspect ratio has on the mechanical and structural properties of granular packings. For grains with maximal symmetry (squares), the stress propagation in the packing localizes forming chainlike forces analogous to the ones observed for spherical grains. This scenario can be understood in terms of stochastic models of aggregation and random multiplicative processes. As the grains elongate, the stress propagation is strongly affected. The interparticle normal force distribution tends toward a Gaussian, and, correspondingly, the force chains spread leading to a more uniform stress distribution reminiscent of the hydrostatic profiles known for standard liquids.
\end{abstract}

DOI: 10.1103/PhysRevLett.103.118001

PACS numbers: $45.70 . \mathrm{Cc}$

Granular packings constitute challenging systems both because of their distinct mechanical properties and due to their technological implications. Despite the fact that granular materials are usually composed of particles with anisotropic shapes, like rice, lentils, or pills, most of the experimental and theoretical studies have been focused on spherical particles [1-4]. However, asphericity, and in general the particle aspect ratio, may give rise to completely different physical scenarios of new phases and critical behaviors. In the last decade, some authors have reported experimental and numerical results about the effect that the particle shape introduces on packing [5], coordination number [6], jamming [7], and stress propagation in granular piles [8]. Nevertheless, the global behavior and the interparticle force distribution of granular systems that are formed by anisotropic particles are still not well understood. Even more relevant, in recent years, there has been a rising awareness that granular materials composed by isotropic grains constitute the exception, rather than the rule [9]. Despite previous efforts, a thorough study of the change of the behavior of these systems as the grains lose their symmetry is still lacking.

In this Letter, we systematically study how the packing properties of rods settling under gravity vary with grain's elongation. We compare quantitatively experimental data with numerical simulations, thus extending the range of aspect ratios covered experimentally. Using established mean-field theories, the interrelation between the morphology and force transmission in granular packings is carefully examined. The analysis of the distributions of particle orientation, cluster size, and contact forces reveals that square grains constitute a peculiar system, which shows qualitatively different properties from elongated grains. In addition, we show that the increase of the particle aspect ratio leads to a distortion of the force chains evidenced by an homogenization of the interparticle normal force distribution function.
Our experimental setup consists of a two dimensional silo (1.1 mm thick, $180 \mathrm{~mm}$ wide and $790 \mathrm{~mm}$ high). The silo is made up of two glass plates separated by two stainless-steel strips of $1.1 \mathrm{~mm}$ thickness so that the granular material is confined in a monolayer between the plates. The bottom of the silo is flat and made of stainless steel. The two types of particles used in this study were monodisperse stainless-steel rods with an aspect ratio $d=2.4$ and $d=5.4$ (i.e., both types had a diameter of $1.0 \mathrm{~mm}$ and lengths of 2.4 and $5.4 \mathrm{~mm}$, respectively). The granular sample was introduced from the top of the silo homogeneously along its whole width at a loading rate of $200 \mathrm{rods} / \mathrm{s}$ for $d=2.4$ and $80 \mathrm{rods} / \mathrm{s}$ for $d=5.4$. Once the particles had settled, a Nikon D40X 10.2 megapixel camera was used to take pictures of a region that covers the whole width of the bottom of the silo up to a height of $110 \mathrm{~mm}$ [Fig. 1(a)]. A custom-made image processing program was used to identify the position of every rod and its orientation $\theta$, defined as the angle between the longer side of the rod and the horizontal direction. The experimental data was obtained by performing 100 silo fillings for each sample. That is to say, we analyzed more than $7 \times 10^{5}$ of the 2.4 rods and $3 \times 10^{5}$ of the 5.4 rods.

For the numerical simulations, we consider a system of identical undeformable rods with aspect ratio $d$, within a rectangular container of height $h$ and width $a=32 d$ (hence, always rescaled to the particle aspect ratio). The boundaries of the system are fixed particles which mimic rigid walls. The particles are continuously added at the top of the system until the prescribed system size $N_{o}=3500$ is reached. For the contact interaction, we use a two dimensional approach, which allows us to cover a wide range of aspect ratios $[10,11]$. When two particles come in contact, their interaction force, $\vec{F}_{c}=\vec{F}^{e}+\vec{F}_{v}$, has two contributions: one elastic, $\vec{F}^{e}$, and one viscous, $\vec{F}_{v}$. Both contributions are decomposed in a component parallel to the direction that joins the centers of mass of the two particles, 


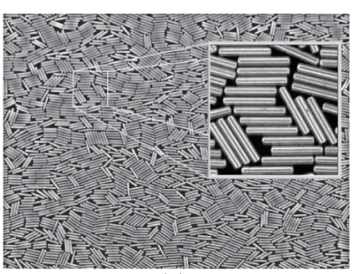

(a)

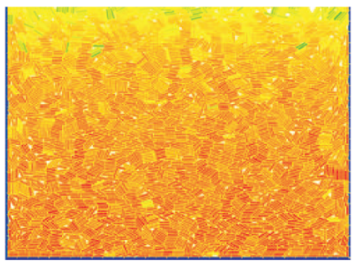

(c)

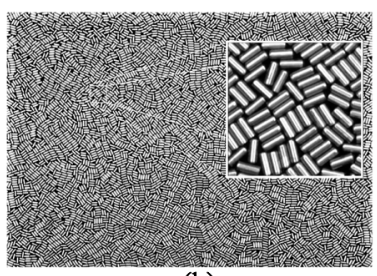

(b)

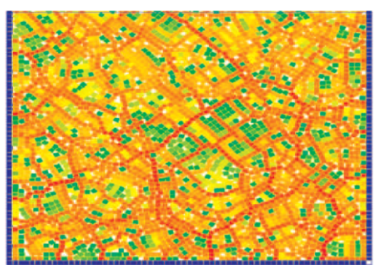

(d)
FIG. 1 (color online). Packings of particles obtained for different aspect ratios. Pictures from the experiment for $d=5.4$ (a) and $d=2.4$ (b) and numerical graphs for $d=5.4$ (c) and squares $d=1$ (d). The color map displays in log scale the local mean value of the contact forces on each particle, normalized by the average value within the whole system.

n, and another component perpendicular to it. The elastic part of the normal contact force, $F_{n}^{e}=-k_{n} A$, is proportional to the overlap area, $A$, between the two particles, while the tangential component, $F_{t}^{e}=-k_{t} \xi$, is proportional to the elastic elongation $\xi$ of a Cundall-Strack's spring [12], where $k_{t}$ stands for the tangential stiffness. The dissipation force is of viscous origin, with components parallel, $F_{n}^{v}=-m_{r} \gamma_{n} v^{n}$, and perpendicular, $F_{t}^{v}=$ $-m_{r} \gamma_{t} \boldsymbol{v}^{t}$, to $\mathbf{n}$. In these expressions, $m_{r}$ represents the reduced mass of the two interacting particles; $\gamma_{n}$ and $\gamma_{t}$ are, respectively, the normal and tangential damping coefficients, while $v^{n}$ and $v^{t}$ are the corresponding components of the relative velocity of the interacting pair. The calculation of the total tangential forces $\vec{T}$ obeys the constrain of the Coulomb condition $\mu \vec{N}$, where $\mu$ is the static friction coefficient and $\vec{N}$ the total normal force. The $2 N_{o}$ equations of motion were integrated using a fifth order predictorcorrector algorithm with an accuracy of order $(\Delta t)^{6}[13]$. The simulations run until the mean kinetic energy is several orders of magnitude smaller than the initial value and the stresses no longer vary with time. In order to achieve a quantitative comparison with the experiments, we have used the parameters $\frac{k_{t}}{k_{n}}=0.1, \quad k_{n}=10^{3} \mathrm{Nm}, \quad g=$ $10 \mathrm{~m} / \mathrm{s}^{2}, \frac{\gamma_{n}}{\gamma_{t}}=3, \gamma_{n}=10^{3} \mathrm{~s}^{-1}, h=1.2 \mathrm{~m}$, and $\mu=$ 0.6 ; only the aspect ratios of the particles have been changed. These values were chosen in line with reasonable estimates of real materials. No qualitative changes were appreciated upon changing the previous values for a wide range of parameter sets.

Figure 1 shows the rod packings obtained experimentally for $d=5.4$ and $d=2.4$, and numerically for $d=5.4$ and $d=1$ (squares). For the numerical results, the local mean value of the contact force, normalized by its average value within the whole system, is displayed as a color image (online) in log-scale. The first result that becomes apparent is that the elongated rods tend to orient with their long axis parallel to the substrate, i.e., perpendicular to the gravity. At the same time, the numerical pictures for squares $(d=1)$ reveal the development of a force network, an intrinsic characteristic of granular solids. In contrast, for the $d=5.4$ rods, the force is transmitted rather more uniformly, and the increasing of the force with depth can be clearly appreciated.

Let us start by presenting the results of the orientation distribution function $f(\theta)$ of particles with different aspect ratios (Fig. 2). Provided that in all the cases the orientation distribution displays an expected symmetry with respect to $\theta=\frac{\pi}{2}$, i.e., the direction of gravity, only the results from $\theta=0$ to $\theta=\frac{\pi}{2}$ are displayed. The good agreement between the experimental and numerical results indicates the predictive accuracy of our numerical simulation. It is clearly seen that long particles lie most probably parallel to the substrate $(\theta=0)$, while the most unlikely position, $\theta=\frac{\pi}{2}$, corresponds to standing rods. This result is in excellent agreement with previous observations of rod packings [14]. As the aspect ratio decreases, there is a shift in the most probable orientation, leading to a peaked distribution at an intermediate orientation. For the squares, the most probable orientation turns out to be $\theta=\frac{\pi}{4}$, i.e., with one of its diagonals in the horizontal direction. It should be noted that we disregarded the contribution of particles located close to the system walls, where rods are aligned preferentially along the wall, as has been reported previously in the case of discs [15].

Although the grain arrangement is a result of the deposition history, we can gain insight in the average grain orientation by considering the quasistatic limit of an array

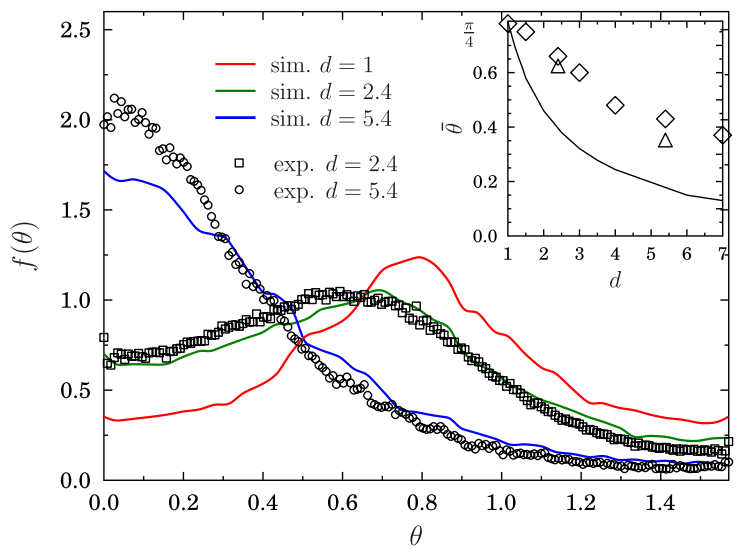

FIG. 2 (color online). Orientation distributions of particles, for several aspect ratios. Inset: Dependence of the average orientation with increasing aspect ratio obtained experimentally $(\diamond)$ and numerically ( $\square$ ). The continuous line represents the analytical prediction for a frictionless deposit. 
of rigid frictionless particles introduced in Ref. [16], where the stress can be fully described in terms of the arrays of contact points [17]. Since in our case gravity tends to align force chains, in mean-field approximation, the probability that a force chain goes through a given particle should be proportional to the relative projection of its sides on the horizontal direction, $S(\theta)$. For a two dimensional rod, $S(\theta)$ is simply given by $S(\theta)=\cos \theta+d \sin \theta$ which is maximized for an orientation $\tan \left(\theta_{p}\right)=\frac{1}{d}$. This implies the expected planar alignment, $\theta_{p}=0$, for long grains $(d \rightarrow$ $\infty)$ and the observed orientation, $\theta_{p}=\frac{\pi}{4}$, for squares $(d=$ 1). In the inset of Fig. 2, the mean rod orientation $\bar{\theta}$ is plotted for different aspect ratios together with the previous prediction for $\theta_{p}$. Clearly, the quasistatic prediction recovers the trend observed in experiments and simulations, but it underestimates the value of the mean orientation, which indicates the relevance of the deposition process and the particle friction in the properties of the final packing [18].

Given the considerable effect that the aspect ratio has on the particle orientation, it followed that we should then look into its impact on the structure formation within the packing. Since the deposits are highly compacted, we analyzed the distribution of clusters (or domains) of aligned particles. Two particles are deemed to belong to the same cluster if they are in contact and their faces are parallel. In our experiments, two rods belong to the same cluster if the distance between any point of their surfaces is smaller than $0.1 \mathrm{~mm}$ and the relative orientation of their faces is smaller than 0.05 degrees [19]. In the simulations, we use the same angular criterion and consider that two particles are in contact when the modulus of the interparticle force is larger than $10^{-6}$ times the average contact force.

Figure 3 shows the experimental and numerical cluster size distribution, $D(s)$, obtained for different aspect ratios.

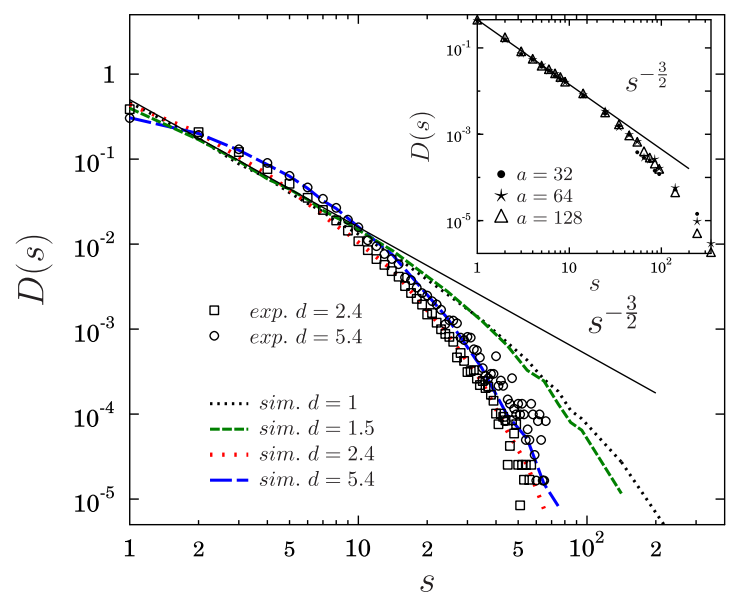

FIG. 3 (color online). Cluster size distribution, $D(s)$, in log-log scale. The inset illustrates results for squares, $d=1$, for several system sizes. The continuous line corresponds to the theoretical algebraic decay, $D(s) \sim s^{-3 / 2}$.
For square particles, small clusters follow an algebraic decay characterized by an exponent $\frac{3}{2}$. This behavior can be understood in the context of aggregation systems with injection [20] or random multiplicative processes [21]. Provided that squares orient preferably at $\theta=\frac{\pi}{4}$ with quite a wide distribution, it seems reasonable that a falling particle aligns only with one of the two grains below it. This simple assumption gives rise to the formation of clusters following a power law with an exponent $\frac{3}{2}$ [20]. The same algebraic distribution is also obtained for the critical $q$ model, where forces are considered to be transmitted asymmetrically through the particle packing [22]. However, for big clusters of squares, a faster decay of the distributions is observed. The inset of Fig. 3 reveals that this decay is not a finite size effect but an evidence that big clusters differ structurally from small ones. As the grains elongate, the asymptotic decay of the cluster distribution deviates from the algebraic decay [23] as a consequence of the vanishing preference for $\pi / 4$ orientation of the particles.

The magnitude of the interparticle forces are easily accessible in the simulations. Figure 4 shows the normal $P\left(F_{n} / \bar{F}_{n}^{l}\right)$ and tangential $P\left(F_{t} / \bar{F}_{n}^{l}\right)$ force distribution of rod packings with different aspect ratios. In order to account for the different mean force values at different height levels, all forces are rescaled with the mean normal force at the corresponding height $\bar{F}_{n}^{l}$. The distribution of normal forces shows an exponential decay for squares and a Gaussian behavior for very elongated rods (see lower-inset of Fig. 4). These results are consistent with what is shown in Figs. 1(c) and 1(d). For squares, the presence of welldefined force chains leads to a wider distribution of contact forces. As the particles elongate the distributions narrow denoting a trend towards uniform force transmission.

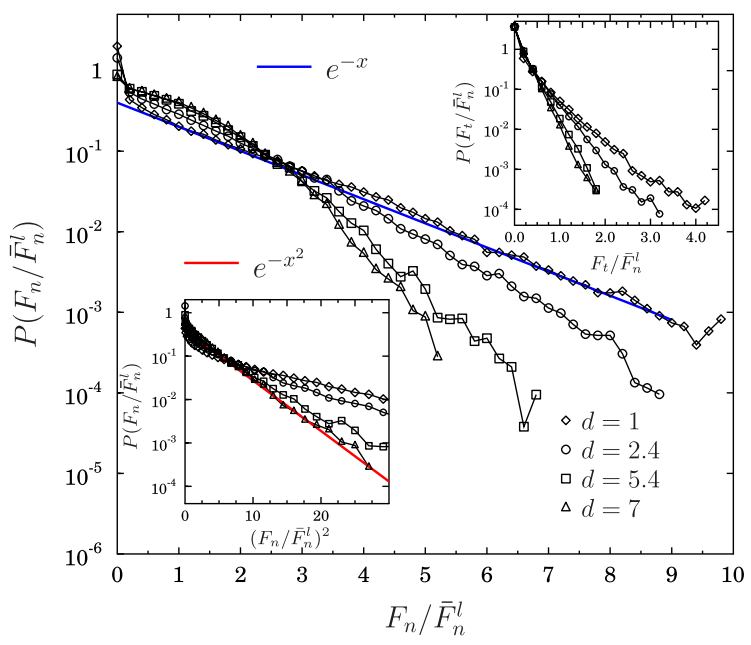

FIG. 4 (color online). Distribution of normal forces for particles with different aspect ratios in semilog scale. The lower inset shows the Gaussian decay for elongated particles. The upper inset shows the distribution of tangential forces. 
Tangential forces turn out to be almost an order of magnitude smaller than normal ones and are distributed exponentially for all the aspect ratios analyzed (see upper inset of Fig. 4). The abrupt decay of the tangential forces, as the particle aspect ratio is increased, indicates their weak relevance in packings involving elongated rods.

Summarizing, we have studied the properties of two dimensional granular packings as their constituents (rods) become symmetric (squares). Elongated particles tend to align parallel to the substrate inducing a quasihomogeneous force transmission in the vertical direction. This scenario is characterized by a Gaussian distribution of interparticle normal forces. As the particles become symmetric, their orientation deviates from the horizontal, leading for the limiting case of squares to an orientation at $\theta=\frac{\pi}{4}$. This results in a heterogeneous structure where well-defined force chains develop while the interparticle normal force distribution evolves into an exponential decay. The correlation between the morphological structure and force distributions suggests the possibility to characterize the stress transmission through appropriate morphological analysis.

Understanding the role of particle elongation in three dimensional deposits remains a relevant open issue. Indeed, although face-to-face oriented particles are expected as in the 2D case [24], it is still not clear the way in which the additional degree of orientation will affect the particle ordering and stress transmission. In any case, the results discussed in this manuscript reveal that most of the structural properties displayed by isotropic granular media in a gravitational field are not easily extrapolated to the case of elongated particles for which new models and theories are necessary.

The Spanish MICINN (Projects FIS2008-06034-C0201/02, FIS2008-04386, Ramon y Cajal Program), and PIUNA have supported this work. We specially thank A. Garcimartín for his useful comments and suggestions concerning this work.

[1] Powders and Grains, edited by R. Garcia-Rojo, H. J. Herrmann, and S. McNamara (Balkema, Leiden, 2005).

[2] C. Liu et al., Science 269, 513 (1995); D. M. Mueth, H. M. Jaeger, and S. R. Nagel, Phys. Rev. E 57, 3164 (1998).

[3] T. S. Majmudar and R. P. Behringer Nature (London) 435, 1079 (2005); H.M. Jaeger, S. R. Nagel, and R.P.
Behringer, Rev. Mod. Phys. 68, 1259 (1996).

[4] I. S. Aranson and L. S. Tsimringy, Rev. Mod. Phys. 78, 641 (2006).

[5] F. X. Villarruel et al., Phys. Rev. E 61, 6914 (2000); A. Donev et al., Science 303, 990 (2004); G. Lumay and N. Vandewalle, Phys. Rev. E 74, 021301 (2006).

[6] J. Blouwolff and S. Fraden, Europhys. Lett. 76, 1095 (2006); A. Wouterse, S. Luding, and A.P. Philipse, Granular Matter 11, 169 (2009).

[7] K. Desmond and S. V. Franklin, Phys. Rev. E 73, 031306 (2006).

[8] I. Zuriguel, T. Mullin, and J. M. Rotter, Phys. Rev. Lett. 98, 028001 (2007); I. Zuriguel and T. Mullin, Proc. R. Soc. A 464, 99 (2008).

[9] M. Mailman et al., arXiv:cond-mat/0812.1234.

[10] F. Lonso-Marroquín and H. J. Herrmann, Phys. Rev. Lett. 92, 054301 (2004); F. Wittel et al., Phys. Rev. Lett. 93, 035504 (2004).

[11] H.J. Tillemans and H.J. Herrmann, Physica A (Amsterdam) 217, 261 (1995); F. Kun and H. J. Herrmann Phys. Rev. E 59, 2623 (1999).

[12] P. A. Cundall and O.D. L. Strack, Géotechnique 29, 47 (1979).

[13] M.P. Allen and D. J. Tildesley, Computer Simulation of Liquids (Oxford University Press, Oxford, 2003).

[14] K. Stokely, A. Diacou, and S. V. Franklin, Phys. Rev. E 67, 051302 (2003).

[15] I. Bartos and I. M. Janosi, Granular Matter 9, 81 (2007); I. Zuriguel, T. Mullin, and R. Arevalo, Phys. Rev. E 77, 061307 (2008).

[16] S.F. Edwards and R.B.S. Oakeshott, Physica A (Amsterdam) 157, 1080 (1989).

[17] R. C. Ball and R. Blumenfeld, Phys. Rev. Lett. 88, 115505 (2002).

[18] A. P. F. Atman et al., J. Phys. Condens. Matter 17, S2391 (2005).

[19] The cluster size distribution is very sensitive to the orientation threshold and almost independent on the distance threshold provided that it is above $0.05 \mathrm{~mm}$ and below $0.5 \mathrm{~mm}$.

[20] H. Takayasu, I. Nishikawa, and H. Tasaki, Phys. Rev. A 37, 3110 (1988); G. Huber, Physica A (Amsterdam) 170, 463 (1991).

[21] C.F. Moukarzel, J. Phys. Condens. Matter 14, 2379 (2002).

[22] S. N. Coppersmith et al., Phys. Rev. E 53, 4673 (1996); J. F. Wambaugh, arXiv:cond-mat/0603314.

[23] The cluster size distribution becomes exponential in $\sqrt{s}$ for very elongated grains.

[24] E. Azéma, F. Radjai, and G. Saussine, Mech. Mater. 41, 729 (2009). 